\title{
Study on acoustic source characteristics of distributed optical fiber acoustic wave monitoring buried natural gas pipeline leakage
}

\author{
Chun Wang ${ }^{1}$, Zan Wang ${ }^{2}$, Jia Zhang ${ }^{3, *}$, and Kelong Yang ${ }^{4}$ \\ ${ }^{1}$ Zhanjiang Branch, CNOOC Ltd, Zhanjiang, Guangdong, 524000, China. \\ ${ }^{2}$ CNOOC Ener Tech-Dilling \& Production Co, Tianjin, 300000, China. \\ ${ }^{3}$ State Key Laboratory of Oil and Gas Reservoir Geology and Exploration, Southwest Petroleum University, Chengdu, Sichuan, 610500, \\ China. \\ ${ }^{4}$ Northwest Sichuan Gas Mine of Southwest Oil and Gas Field Branch, CNPC, Mianyang, Sichuan, 621700, China.
}

\begin{abstract}
In order to study the leakage of buried natural gas pipeline caused serious environmental pollution and human casualties, the acoustic propagation characteristics of buried natural gas pipeline leakage monitored by distributed optical fiber were studied. At present, the research on the leakage of buried pipeline mainly focuses on the propagation of sound waves along the pipe wall, while the study on the propagation of sound waves in the soil is still lacking. The acoustic attenuation of acoustic wave propagation in soil by the size of leakage hole and leakage pressure is studied, and the evolution process of acoustic wave in soil is revealed. The conclusion is that the acoustic source of buried natural gas pipeline leakage belongs to broadband noise, and the acoustic energy of leakage is prominent in the low frequency band of $15 \mathrm{kHz}$. The lower frequency, the higher sound pressure level. The oscillation of the sound pressure level attenuates with the increase of frequency. Fiber optic monitoring of buried natural gas pipeline leakage early warning provides theoretical support for the conclusion. The sound pressure level in low frequency band is of great significance for buried pipeline leakage monitoring.
\end{abstract}

\section{Introduction}

Oil and gas pipeline network is the national vein of national energy and economic development. The construction and development of the pipeline network provides the strategic overall situation of long-term, stable, economic and safe energy guarantee for national economic construction [1-3]. In a long-term and complex environment, pipelines are prone to fracture, medium corrosion, environmental corrosion and defects during operation [4]. Due to the variety of transport media, the variety of leakage forms. A reliable technology has not yet been developed to solve the leakage problem. As a new technology, distributed optical fiber monitoring can carry out remote data acquisition and real-time monitoring, and can be used for leak monitoring and alarm prediction of natural gas pipelines [5]. Among them, distributed optical fiber acoustic sensing technology is based on coherent rayleigh scattering principle. When the pipeline leaks, there is pressure difference between inside and outside the pipeline, and the vibration is generated when the gas overflows from the leakage hole. It spreads around in the soil, and due to the diffusion of acoustic waves, the scattering of acoustic waves by the soil skeleton and the absorption and attenuation of acoustic energy by the soil. When acoustic wave travels to the optical fiber to be measured, it causes the optical fiber to strain, resulting in the corresponding change of optical fiber refractive index, and will immediately send out a warning, so as to realize the realtime monitoring of the oil and gas pipeline without blind area.

There are a lot of studies on leakage acoustic waves in the world. Ong [6] proposed a simple and low-cost acoustic vibration sensor based on a single-mode fiber with micro-curved coating, sensor is adopted macro bending loss as the sensing mechanism to detect low frequency leakage. Stajanca [7] used fiber distributed acoustic sensor to detect gas vibration, and realized the detection of gas leakage in a pipeline with a pressure of 8 atmospheres. Bassim [8] studied the attenuation of acoustic wave in transmission and proved that the attenuation of high-frequency signal is greater than that of low-frequency signal. Gao [9] collected the vibration information of the pipeline wall through the fiber Bragg grating sensing system. The results show that obvious vibration wave can be generated at the moment of gas pipeline leakage, and the vibration acceleration of the pipeline wall near the leakage hole increases. Wang [10] realized the precise monitoring and positioning of gas pipeline leakage in the simulated pipeline leakage experiment field by using the sensor. Li [11] carried out leakage experiments of different shapes of leakage orientations. The results show that there is outburst

Corresponding author: zhangiiaswpu@163.com 
frequency response in the range of $50 \mathrm{kHz}$ to $52 \mathrm{kHz}$ for all the different leakage sources. Yan [12] obtained the law that the mean value of sound pressure level increases with the increase of the internal pressure. It is obtained that the mean sound pressure level increases with the increase of internal pressure and leakage hole.

Through the above literature review, it can be seen that there have been a lot of achievements in pipeline gas leakage. And more based on the flow rate and pressure and other parameters of monitoring and sound propagation along the wall. However, there is a lack of research on acoustic wave propagation and propagation mode in porous soil after buried natural gas pipeline leakage. In this paper, the finite element method (FEM) is used to numerically simulate the sound field propagation of gas leakage from buried natural gas pipeline, and the distribution law of the sound field in soil when gas leakage occurs is obtained, which provides a theoretical basis for the research and development of distributed optical fiber acoustic detection technology and the field application.

\section{Governing equation}

Pipeline leakage process is the fluctuating pressure field change process generated by turbulent process, although the formation mechanism of the sound source is complex, the leakage process still satisfies the three basic equations of the tiny sound waves in an ideal fluid, the equation of mass conservation, the equation of motion, the equation of state $[13,14]$. The liquid density is $\rho$, the velocity moment is $u$, the time is $t$, the pressure is $p$, and the specific heat ratio is $\gamma$. The equation is expressed as follows,

$$
\begin{gathered}
\frac{\partial \rho}{\partial t}+\operatorname{div}(\rho u)=0 \\
\rho \frac{d u}{d t}+\operatorname{grad}(p)=0 \\
\frac{p}{p_{0}}=\left(\frac{\rho^{\prime}}{\rho_{0}}\right)^{\gamma}
\end{gathered}
$$

The three-dimensional acoustic wave equation can be obtained from the above basic equations of fluid motion.

$$
\begin{gathered}
\frac{1}{c_{0}^{2}} \frac{\partial^{2} p}{\partial t^{2}}-\nabla^{2} p=0 \\
\nabla^{2}=\frac{\partial^{2}}{\partial x^{2}}+\frac{\partial^{2}}{\partial y^{2}}+\frac{\partial^{2}}{\partial z^{2}}
\end{gathered}
$$

$c_{0}$ is the propagation velocity, the two-dimensional acoustic wave equation can be expressed as,

$$
\frac{\partial^{2} p}{\partial t^{2}}-c_{0}^{2} \frac{\partial^{2} p}{\partial x^{2}}-c_{0}^{2} \frac{\partial^{2} p}{\partial y^{2}}=0
$$

Equation (6) is the general law followed by sound wave propagation and can be used as the governing equation to describe wave fluctuation, $x$ and $y$ are spatial coordinates, $t$ is time, and $p$ is sound pressure.

In the process of pipeline leakage, the leakage is not only the main sound source of the jet sound, but also the non-uniform airflow refracts and interferes with the sound wave. Therefore, considering the influence of the jet shear layer on the sound propagation, the acoustic wave equation (6) can be written into the following equation.

$$
\frac{\partial^{2} p}{\partial t^{2}}-\left(c_{0}+u_{x}\right)^{2} \frac{\partial^{2} p}{\partial x^{2}}-\left(c_{0}+u_{y}\right)^{2} \frac{\partial^{2} p}{\partial y^{2}}=0
$$

Equation (7) is the acoustic wave governing equation after loading velocity disturbance, where $U_{\mathrm{x}}$ is the velocity of the disturbance in the $x$-direction at a certain position, and $U_{\mathrm{y}}$ is the velocity of the disturbance in the $y$-direction at a certain position.

\section{Numerical model}

A two-dimensional buried natural gas pipeline leakage model was established. The calculation model consists of a gas pipeline, leakage hole and soil, as shown in figure 1. Calculated domain length $\mathrm{L}=5000 \mathrm{~mm}$, height $\mathrm{H}=3200 \mathrm{~mm}$, pipe diameter $150 \mathrm{~mm}$, leakage hole is a conventional round hole, equivalent diameter $1 \mathrm{~mm}$. And the optical fiber is located at $300 \mathrm{~mm}$ above the pipe. The leakage hole is arranged as the pressure inlet and the surface is the pressure outlet. The radiation boundary condition is used to obtain better simulation effect. Cartesian coordinate system, navier-stokes equations (N$\mathrm{S})$ and $\mathrm{k}-\varepsilon$ turbulence model for compressible high velocity fluid are used as mathematical models. For the turbulent sound source of gas, it can be regarded as the result of the interaction of monopole, dipole and quadrupole, among them, the quadrupole sound source is the sound generated by turbulence due to a large number of vortices in the high-speed air flow. The soil property parameters in a certain area are shown in Table 1.

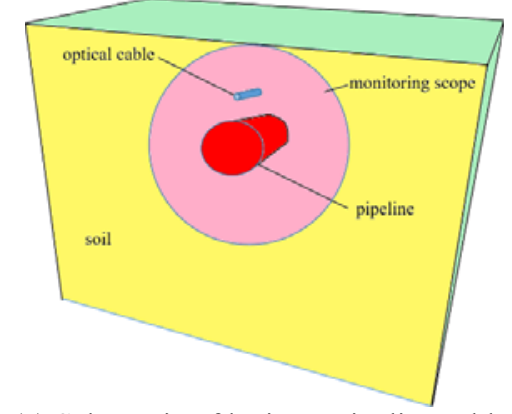

(a) Schematic of laying a pipeline cable.

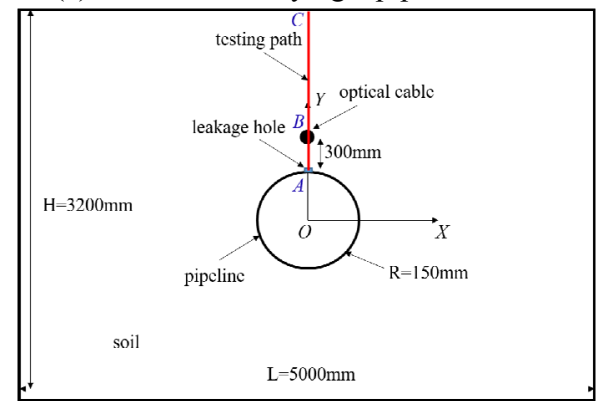

(b) Monitoring points of sensors.

Fig. 1. Gas leakage calculation model of buried pipeline. 
Table 1. Physical parameters of soil.

\begin{tabular}{|c|c|c|c|}
\hline $\begin{array}{c}\text { Density } \\
\left(\mathbf{k g} / \mathbf{m}^{3}\right)\end{array}$ & $\begin{array}{c}\text { Specific heat } \\
\text { capacity } \\
\mathbf{J} /(\mathbf{k g} \cdot \mathbf{K})\end{array}$ & $\begin{array}{c}\text { Heat conductivity } \\
\text { coefficient } \\
\mathbf{w} /(\mathbf{m} \cdot \mathbf{K})\end{array}$ & $\begin{array}{c}\text { Soil } \\
\text { porosity }\end{array}$ \\
\hline 1925 & 2180 & 1.4 & 0.33 \\
\hline
\end{tabular}

Considering the complexity and precision of the solution, the effect of grid independence is eliminated. The model is divided into unstructured grids, and the overall grid quality is above 0.8 .

\section{Numerical simulation analysis}

Pipeline leakage will produce a jet sound source, the two most important factors that affect the jet sound source are the leakage pressure and leakage hole. Therefore, this paper analyzes the leakage sound source characteristics of the pipeline under different leakage hole and different leakage pressures.

\subsection{Influence of different leakage hole}

Based on the acoustic governing equation, the leakage state of the leakage orifice with diameter $\mathrm{D}=1,3,5$ and $7 \mathrm{~mm}$ is numerically analyzed with the internal pressure $\mathrm{P}=4 \mathrm{MPa}$ unchanged. The simulation results of the corresponding sound field sound pressure level (SPL) are shown in figure 2 below.

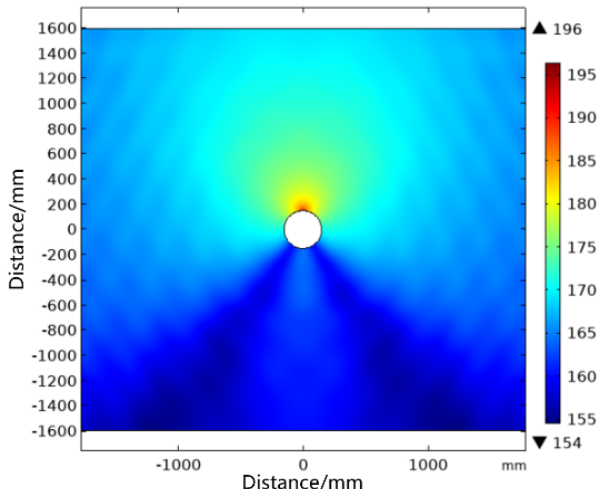

(a) $\mathrm{D}=1 \mathrm{~mm}$

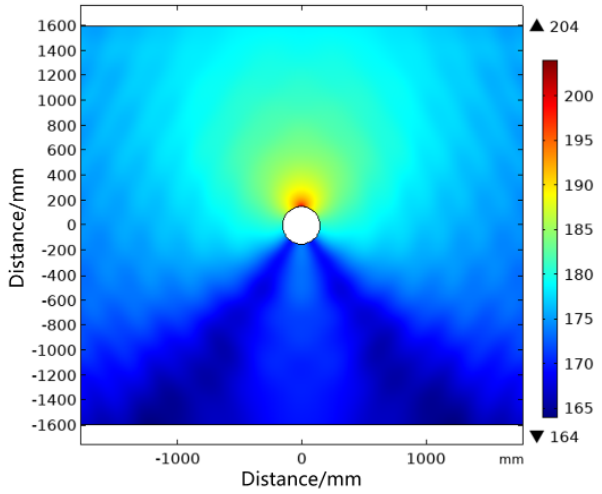

(b) $\mathrm{D}=3 \mathrm{~mm}$

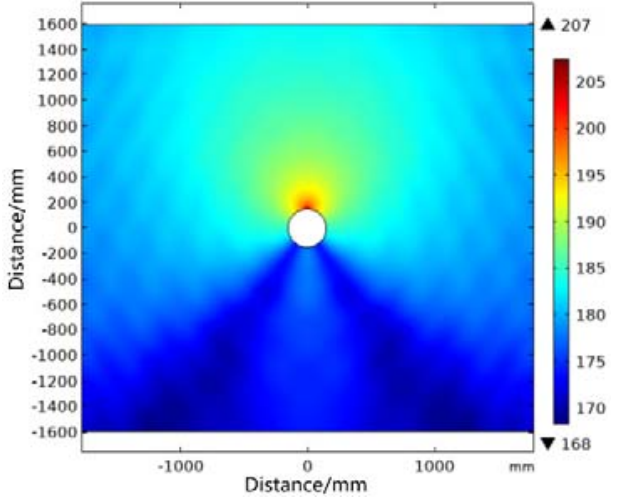

(c) $\mathrm{D}=5 \mathrm{~mm}$

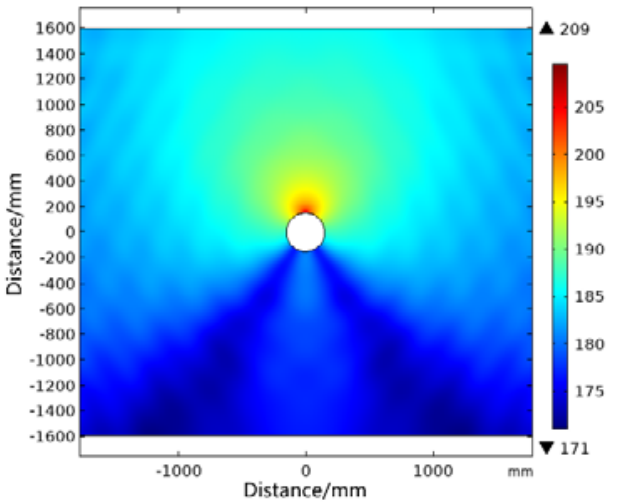

(d) $\mathrm{D}=7 \mathrm{~mm}$

Fig. 2. Distribution of total sound pressure levels under different leakage hole.

As can be seen from Fig. 2, when the internal pressure in the pipeline is constant, the sound field changes with the change of the leakage hole. When the leakage hole is $1 \mathrm{~mm}$, the maximum total sound pressure level reaches $196 \mathrm{~dB}$, and when the leakage hole is $7 \mathrm{~mm}$, the maximum total sound pressure level reaches $209 \mathrm{~dB}$. That is, with the increase of the diameter of the leakage orifice, the mass velocity of the leakage medium increases, and the intensity of the excited sound field also increases. In addition, due to the small acoustic waves at the leakage hole, the acoustic waves are superimposed upon each other after the reflection of the two walls of the leakage hole, so the sound pressure at the leakage hole is far greater than the far-field sound pressure intensity.

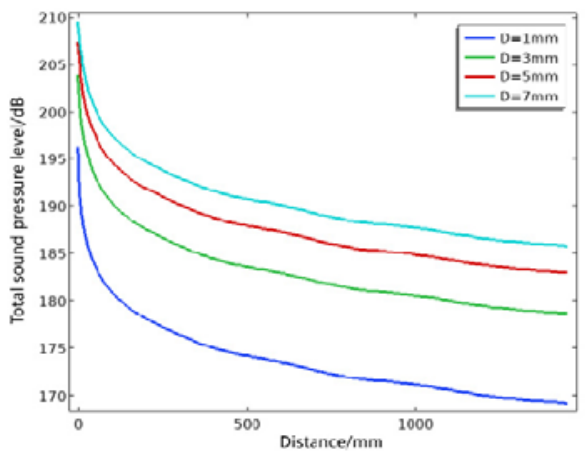

Fig. 3. SPL distribution on path AB. 


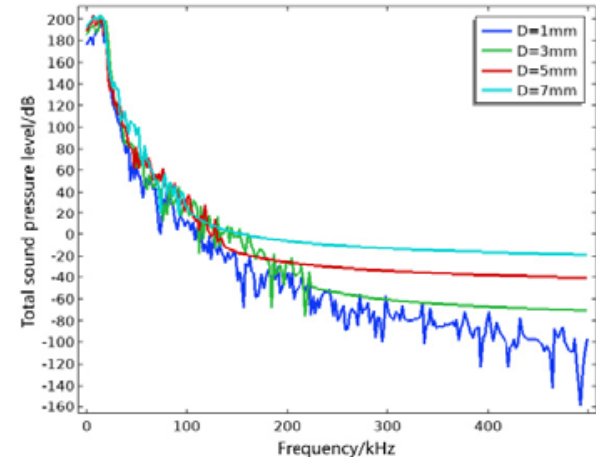

Fig. 4. SPL distribution on $y=300 \mathrm{~mm}$.

It can be seen from figure 3 that the farther away from the leak, the smaller the total sound pressure level. The larger the leak, the slower the attenuation of sound pressure. Taking the monitoring point at $y=300 \mathrm{~mm}$ on the path as the observation object. Analyze the total sound pressure level distribution at $y=300$ under the same leakage hole. Frequency domain characteristics of sound pressure levels of sound sources corresponding of leakage hole are analyzed. As can be seen from figure 4, the acoustic wave radiated to the far field of the soil is enhanced locally at the leakage hole. When the strength of the leakage jet stream weakens, the acoustic wave intensity gradually decreases. The larger leakage hole is, the more the total sound pressure level is to be stable. In particular, when the frequency is $15 \mathrm{kHz}$, the SPL is the highest, the maximum is $198.65 \mathrm{~dB}$, and the SPL drops rapidly between $15 \mathrm{kHz}$ and $50 \mathrm{kHz}$. When the SPL is $100 \mathrm{kHz}$, the total SPL drops to 0 . On the whole, the sound pressure at $y=300$ gradually decreases with the increase of the leakage hole, which also indicates that the intensity of the leakage sound field also decreases. In other words, the smaller the leakage hole is, the more obvious the acoustic superposition of the leakage hole is, and the farther the sound pressure spreads.

\subsection{Influence of different leakage pressure}

The leakage hole $\mathrm{D}=1 \mathrm{~mm}$ of the pipeline is kept unchanged, and the leakage state of the pipeline is numerically simulated for the leakage pressure $\mathrm{P}=2,4,8$ and $12 \mathrm{MPa}$. The simulation results of the corresponding sound pressure level of the sound field are shown in figure 5 below.

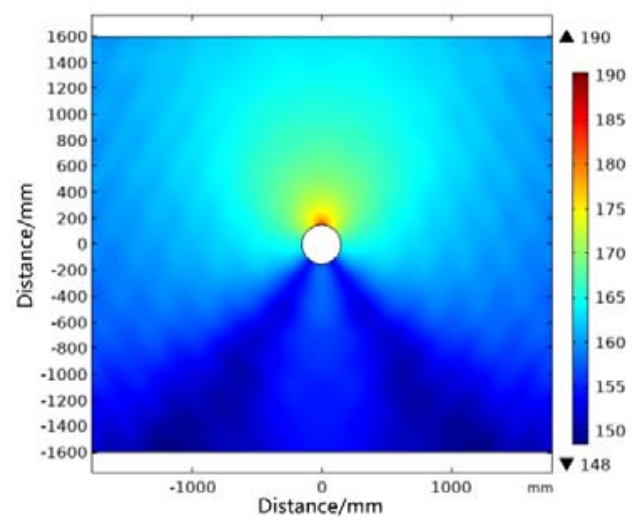

(a) $\mathrm{P}=2 \mathrm{MPa}$

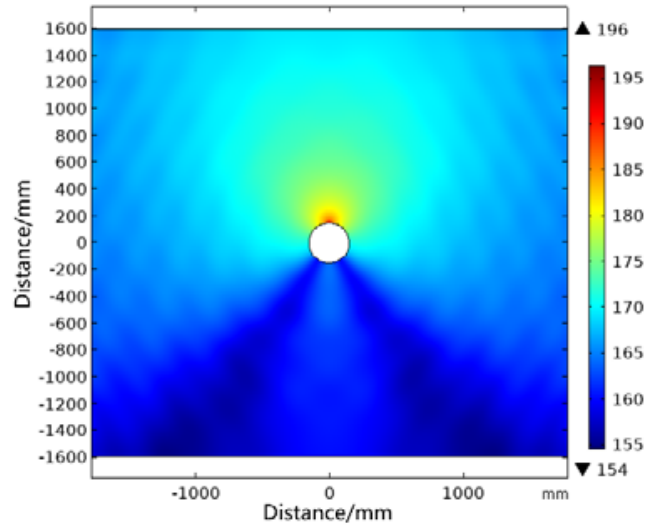

(b) $\mathrm{P}=4 \mathrm{MPa}$

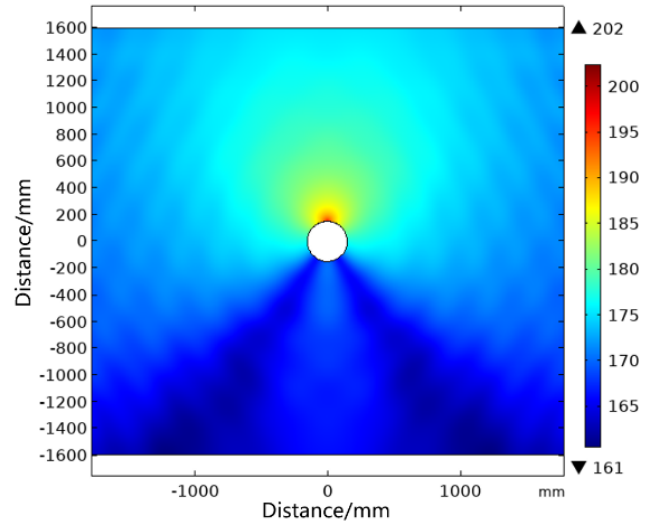

(c) $\mathrm{P}=8 \mathrm{MPa}$

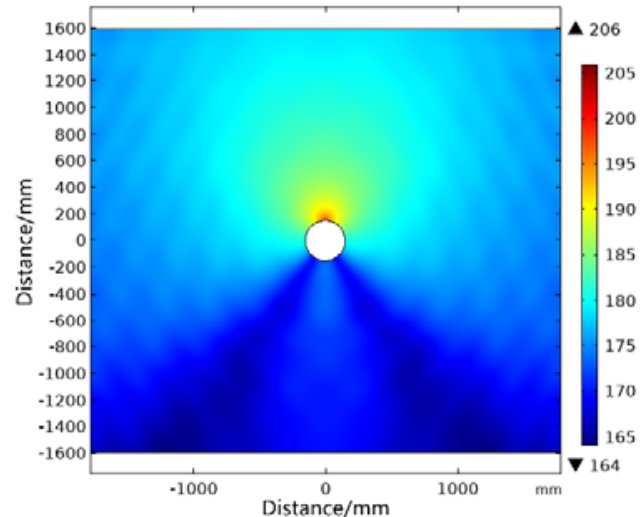

(d) $\mathrm{P}=12 \mathrm{MPa}$

Fig. 5. Distribution of total sound pressure levels under different leakage pressures.

Figure 5 shows the distribution of the total sound pressure level of leakage under different leakage pressures. Similar to the influence of different leakage hole on the sound source characteristics, the sound source of pipeline leakage radiates to the porous soil in all directions. The acoustic pressure intensity of the leakage sound wave is higher only at leakage hole and the local area around the leakage hole, while the far-field acoustic wave is weaker than that at leakage hole. Although the pressure $\mathrm{P}$ in the pipe keeps increasing, the distribution of sound field is basically unchanged, but the overall sound intensity and the intensity of the leakage sound source increase accordingly. 


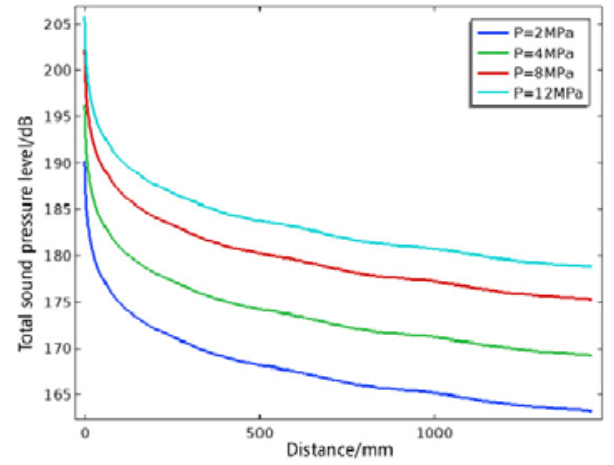

Fig. 6. SPL distribution on path $A B$.

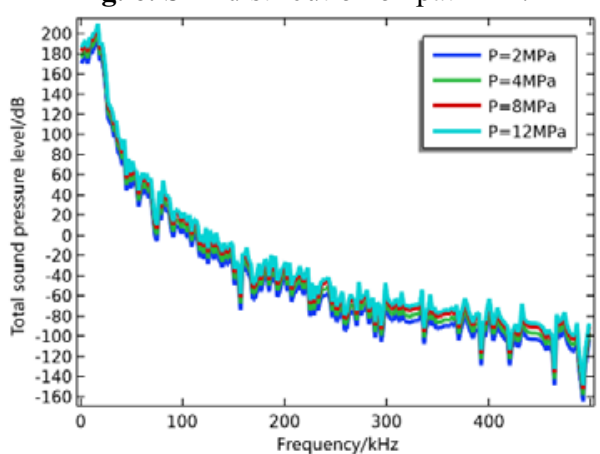

Fig. 7. SPL distribution on $y=300 \mathrm{~mm}$.

It can be seen from figure 6 that the sound pressure of the leakage jet increases with the increase of the pressure $\mathrm{P}$, indicating that the pressure in the pipeline has a great influence on the sound intensity generated by the leakage. It can be seen from figure 7 , the total sound pressure level decreases with the increase of the leakage pressure $\mathrm{P}$ at the monitoring point $\mathrm{y}=300$. In particular, the SPL is the highest when the frequency is $15 \mathrm{kHz}$ and the maximum is $210.22 \mathrm{~dB}$. Similarly, the SPL drops rapidly between $15 \mathrm{kHz}$ and $50 \mathrm{kHz}$. When the SPL is greater than $50 \mathrm{kHz}$, the total SPL drops slowly, and when the SPL is $100 \mathrm{kHz}$, the total SPL drops to $0 \mathrm{~dB}$. Therefore, the sound pressure level in the ultra-low frequency segment is of great significance to the detection and location of gas pipeline leakage by acoustic wave method.

\section{Acknowledgments}

This work is supported by the key research and development projects of Sichuan province (2020YFG0180), national natural science foundation of China (51974271), and Xinjiang oilfield company project of PetroChina (Safety risk analysis and countermeasure study of natural gas cryogenic temperature alternating condition).

\section{References}

1. Lang X.M, Li P, Cao J.T, et al. Research progress of leak detection and location technology of oil and gas pipeline in long distance transportation[J]. Control engineering, 2018, 25(004): 621-629.

2. Lu H.F, Wu X.N, Tom I, et al. Present situation and enlightenment of foreign natural gas pipeline detection technology[J]. Natural gas industry, 2018(2): 103-111.

3. Adegboye M.A, Fung W.K, Karnik A. Recent advances in pipeline monitoring and oil leakage detection technologies: principles and approaches $[\mathrm{J}]$. Sensors, 2019, 19(11): 2548.

4. Zaman D, Tiwari M.K, Gupta A.K, et al. A Review of Leakage Detection Strategies for Pressurised Pipeline in Steady-State[J]. Engineering Failure Analysis, 2019: 104264.

5. Liu J.X. Research progress of $\emptyset$-OTDR distributed optical fiber sensing monitoring technology[J]. Advances in lasers and optoelectronics, 2013, 50(08): 199-204.

6. Ong K.S, Png W.H, Lin H.S, et al. Acoustic vibration sensor based onmacro-bend coated fiber for pipeline leakage detection. IEEE, 2017:167-171.

7. Pavol S, Sebastian C, Tobias H, et al. Detection of Leak-Induced Pipeline Vibrations Using Fiber-Optic Distributed Acoustic Sensing[J]. Sensors, 2018, 18(9): 2841.

8. Bassim M.N , Tangri K. Leak Detection in Gas Pipelines Using AcousticEmission [R]. Edmonton Alberta, Canada: International Conference, 1984.

9. Gao W.N, Liu Y, Li D.K, et al. Research on gas pipeline leakage based on optical fiber monitoring technology[J]. China Safety Science Journal, 2019, 29(08): 67-72.

10. Wang C, Liu Q.W, Chen D, et al. Pipeline leakage monitoring based on distributed optical fiber acoustic sensing[J]. Journal of the optical, 2019, 39(10): 119-125.

11. Li Y.B, Jing W.X, Xue S, et al. Influence of the shape of the leakage hole on the characteristics of the leakage sound source of the gas pipeline $[\mathrm{J}]$. The Chinese test, 2020, 46(11):139-145.

12. Yan C.W, Han B.K, Bao H.Q, et al. Study on acoustic source characteristics of gas pipeline leakage[J]. acoustic technique, 2017, 36(2): 110-115.

13. Ma D.Q. Fundamentals of modern acoustics[M]. Beijing: science press, 2005: 249-265.

14. Wang B. Numerical simulation and experimental study of sound field in valve leakage[D]. Daqing: Daqing Petroleum Institute, 2007: 55-58. 